\title{
An Analogy Between the Properties of Light and Properties of Vortex-Wave Process in the Medium Similar to Superfluid ${ }^{3} \mathrm{He}-\mathrm{B}$
}

\author{
Liudmila B. Boldyreva* \\ The State University of Management \\ *Corresponding author: boldyrev-m@yandex.ru
}

Received December 07, 2014; Revised February 05, 2015; Accepted February 09, 2015

\begin{abstract}
Characteristics of three types of photon used in the descriptions of optical experiments demonstrating the properties of light are discussed: C-photon - a quasi-monochromatic electromagnetic radiation localized in space, M-photon - a hypothetical elementary particle of the light field, and Q-photon - an objective entity corresponding to the Fock state of the light field. Some properties of superfluid 3He-B are analyzed and equations are derived describing a vortex-wave process in the medium similar to superfluid $3 \mathrm{He}-\mathrm{B}$. The comparison of the properties of light and those of vortex-wave process in the medium similar to superfluid 3He-B shows that there is an analogy between the properties of light and the properties of the process. The analogy found allows one to offer a physical model describing such properties of light as the constancy of its speed in inertial frames of reference, and quantum correlations of photons.
\end{abstract}

Keywords: photon, speed of light, quantum correlations, spin supercurrent, superfluid physical vacuum, vortexwave process

Cite This Article: Liudmila B. Boldyreva, "An Analogy Between the Properties of Light and Properties of Vortex-Wave Process in the Medium Similar to Superfluid ${ }^{3}$ He-B." International Journal of Physics, vol. 3, no. 2 (2015): 74-83. doi: 10.12691/ijp-3-2-5.

\section{Introduction}

According to [1], there are three types of photon used in the descriptions of optical experiments demonstrating the properties of light.

The C-photon is a classical wave packet, that is, a quasimonochromatic electromagnetic radiation localized in space, carrying a quantum of energy $\hbar \omega_{p h}$, where $\omega_{p h}$ is the central frequency of the radiation spectrum. The wave packets are described by the equations of classical electrodynamics, i.e. by Maxwell's equations. Preservation of the plane of electric component of photon is evidence of gyroscopic properties of the latter. The "corpuscular" properties of the C-photon reveal themselves only at the moment of its detection.

It is noteworthy that the representation of each individual photon as a C-photon, i.e. as a wave packet of definite shape and polarization, is inconsistent with the quantum description of quantum correlated photons. One of the main properties of C-photon is the speed of its propagation in a vacuum. The principal theory describing the results of the experiments on determining the speed of light is special relativity. The special theory of relativity postulate of the constancy of the speed of light is as follows [2]: in any inertial frame of reference, light propagates isotropically, independent of the motion of its source, and the speed of light is equal to the absolute constant $c$. The experiment performed at CERN, Geneva, in 1964 [3] is considered the most convincing evidence of the postulate of the constancy of the speed of light. In this experiment the speed of $6 \mathrm{GeV}$ photons produced in the decay of very energetic neutral pions was measured. The pions were produced by bombardment of a berylium target by $19.2 \mathrm{GeV}$ protons and had speeds (inferred from measured speeds of charged pions produced in the same bombardment) of $0.99975 \mathrm{c}$. Within experimental error, it was found that the speed of the photons emitted by the extremely rapidly moving source was equal to $c$. If the observed speed is written as $c^{\prime}=c+k^{\prime} v$, where $v$ is the speed of the source, the experiment showed $k^{\prime}=(0 \pm 1.3) \cdot 10^{-4}$. It is noteworthy that the speed of the photons in the experiments was determined relative to the setup placed on the Earth, that is, in the reference frame connected with the earth.

The M-photon is a hypothetical elementary particle of the light field generating a pulse at the output of the photodetector. Although there is no more rigorous definition of the M-photon in the framework of any consistent theory, the photon as a particle (with the wave properties characteristic of the particle) is treated in a lot of optical studies. The introduction of the M-photon makes it possible to provide a casual description of an individual event: the generation of a single pulse at the detector output. Here, it is assumed that any radiation field 
consists of a set of almost independent photons with definite a priori features to be revealed after a time. Note that the first corpuscular models of the light field consisting of elementary particles, the quanta, were developed after A. Compton's experiments on the X-ray scattering (1922) [4]. The observed change in the frequency of the scattered radiation could be explained by elastic collision of an electron and a particle possessing energy $\hbar \omega_{p h}$ and momentum $\hbar \omega_{p h} / c$. M-photon has the angular nomentum equal to $\hbar$. The so-called kinetic mass $m_{p h}$ of photon has been introduced as well: $m_{p h}=\hbar \omega_{p h} / c^{2}$.

The Q-photon is an objective entity corresponding to the Fock state of the light field with $n=1$ or a superposition of such states with nearly equal energies. This definition can be made in terms of the standard quantum theory of light. Essentially, Q-photon was introduced for description of quantum correlations of photons.

Quantum correlations belong to the category of phenomena that are collectively called "quantum nonlocality". The essence of the phenomena can be described using the following example. Let two quantum entities, e.g. photons $\boldsymbol{a}$ and $\boldsymbol{b}$ at the output of a beam splitter, which are described by the same wave function at the initial moment of time, move in different directions (Figure 1): photon $\boldsymbol{a}$ is directed, depending on the position (1 or 2 ) of switch $P$, either towards detector $A_{1}$ or detector $A_{2}$; photon $\boldsymbol{b}$ is directed towards detector $B$.

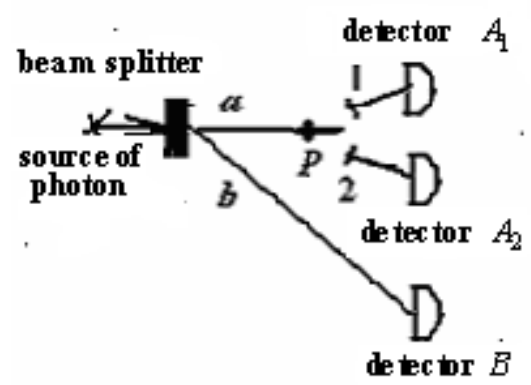

Figure 1. Photon $a$ arrives at either detector $A_{1}$ or detector $A_{2}$ depending on the position of switch $P$ (1 or 2 ), photon $b$ arrives at detector $B$

Since in quantum mechanics any measurement is a reduction of wave function, two different measurements of photon $\boldsymbol{a}$ may result in two different states of photon $\boldsymbol{b}$, that is, the properties of photon $\boldsymbol{b}$ will depend on the interaction of photon $\boldsymbol{a}$ with the detector.

According to experimental data, quantum correlations take place:

1. Independent of the distance between the detectors; this was established over distances of $10 \mathrm{~km}$ [5].

2. Not only between specially prepared photons obtained from the same light source, but also between photon beams of the same frequency, emitted by different sources. This is supported by the fact that overlapping photon beams of the same frequency emitted by different lasers can interfere with each other and thus be coherent [1].

3. In the case of different times of traveling of the photons from their sources to the detectors, that is, when one of the photons has reached its detector and the other one is still en route [6].
Note: this property does not allow the Copenhagen interpretation to be applied to quantum correlations. The Copenhagen interpretation rejects the possibility of endowing quantum entities with a priory properties; the following statement is ascribed [1] to Niels Bohr: "A quantum phenomenon may be considered a phenomenon if only it is a measurement event." However, with different optical paths of photons, in the Copenhagen interpretation a quantum correlation takes place between the detected photon and something which has not yet been detected, i.e. something nonexistent.

4. The features of the process determining quantum correlations can be seen using the example of two-photon interference [1,7]. Let us consider the interference of two beams of light. Figure 2 shows a diagram of experimental setup, where both beams undergo phase delays. Fields $a_{1}$ and $a_{2}$ of frequency $\omega_{a}$ and also fields $b_{1}$ and $b_{2}$ of frequency $\omega_{b}$ are mixed by respective beam splitters.

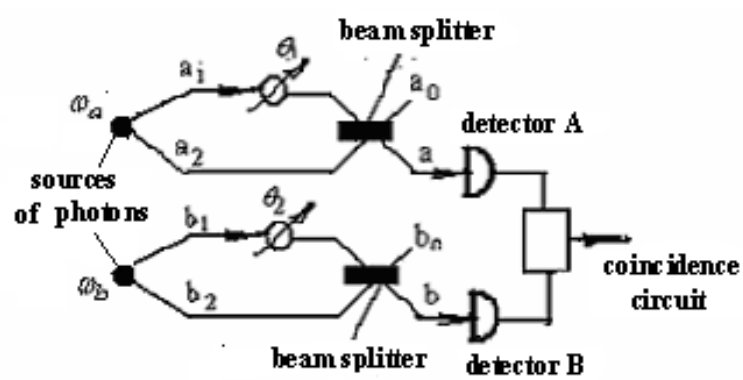

Figure 2. Diagram of a four-mode intensity interferometer: $a_{1}, a_{2}, b_{1}$, $b_{2}$ are input beams of light; $\theta_{1}$ and $\theta_{2}$ are phases of delay of light beams. The detectors and coincidence circuit measure the correlation of intensities

The detectors and coincidence circuit measure the correlation of intensities. The observed correlation and anticorrelation of intensities of output fields $a$ and $b$ depend periodically on phase delays $\theta_{1}$ and $\theta_{2}$. Depending on the statistics of incident beams of light, two types of interference may take place: one with phase $\theta_{1}-\theta_{2}$, the other with phase $\theta_{1}+\theta_{2}$. The first interference is referred to as classical interference (the Hanbury Brown and Twiss intensity interference [7]), the second one is quantum, the so called two-photon interference. Under definite conditions both types of interference are a result of conversion by beam splitters of fluctuations of phases of input fields into fluctuations of intensities of output fields. What are the conditions? Let fields $a_{k}$ and $b_{k}(k=1,2)$ have constant unit amplitudes and respective phases $x_{k}(t)$ and $y_{k}(t)$ which drift with time $t: a_{k}(t)=\exp \left[-i x_{k}(t)\right], b_{k}(t)=\exp \left[-i y_{k}(t)\right]$. The condition of the Hanbury Brown and Twiss interference of intensities is determined by equalities: $x_{1}=y_{1}$ and $x_{2}=y_{2}$. The condition for two-photon interference is as follows: $x_{1}+y_{1}=x_{2}+y_{2}$, that is, for the changes in the phase of field $a, \Delta x$, and field $b, \Delta y$, the following is valid:

$$
\Delta x=-\Delta y
$$

These two conditions can be respectively called the condition of correlation and the condition of anticorrelation of phases. 
5. Theoretically, the results of experiments on quantum correlations can be interpreted without ignoring the cause and effect relationships by introducing unknown "superluminal" forces between the correlated photons.

It is shown in this paper that if the physical vacuum had the properties of superfluid ${ }^{3} \mathrm{He}-\mathrm{B}$ (hereafter referred to as the superfluid physical vacuum - SPV), a vortex-wave process whose properties are similar to the properties of photon given above could propagate in such a vacuum.

The validity of endowing the physical vacuum with the properties of superfluid ${ }^{3} \mathrm{He}-\mathrm{B}$ is substantiated in a number of works. For example the superfluid properties of physical vacuum explain the dissipation free motion of celestial bodies, such as the planets of the solar system [8]. In Refs. [9-11] an analogy is shown between some properties of superfluid ${ }^{3} \mathrm{He}-\mathrm{B}$ and gravitational properties of space, and in Refs. [12-17] an analogy is shown also between some properties of superfluid ${ }^{3} \mathrm{He}-\mathrm{B}$ and the effects of biologically active substances in ultra-low doses and low-intensity physical factors (including the cavity structure effect) on biological systems.

\section{Some Properties of ${ }^{3} \mathrm{He}-\mathrm{B}$}

(For properties 1-11 the references used are [18,19], for properties 12-13 the references are [20,21,22].)

1. In the unperturbed state superfluid ${ }^{3} \mathrm{He}-\mathrm{B}$ is a homogeneous and isotropic medium.

2. In superfluid ${ }^{3} \mathrm{He}-\mathrm{B}$, as in any other superfluid, there is no shear viscosity.

3. Quantized vortex lines are created in superfluid ${ }^{3} \mathrm{He}-$ $\mathrm{B}$ under rotation, i.e. vortex lines in which the angular momentum of paired ${ }^{3} \mathrm{He}$ atoms equals $n \hbar$, where $\hbar$ is Planck's constant, $n=1,2 \ldots$

4. The quantized vortices produced in ${ }^{3} \mathrm{He}-\mathrm{B}$ do not diffuse in the stationary state.

5. The magnetization of the vortex core along the vortex axis takes place in superfluid ${ }^{3} \mathrm{He}-\mathrm{B}$, i.e. spin polarization of superfluid arises in the vortex core. This means that the Barnett effect takes place in the superfluid: the angular momentum of paired ${ }^{3} \mathrm{He}$ atoms that constitute the vortex can be transferred to spins of the atoms, which process is more pronounced in the core. The existence of the Barnett effect in superfluid ${ }^{3} \mathrm{He}-\mathrm{B}$ agrees also with the experimental findings that the vortices having oppositely oriented spins of the constituent atoms are characterized by mutually opposite velocity circulation directions (in other words, the spin-up condensate circles in the direction opposite to that of the spin-down condensate).

6. In superfluid ${ }^{3} \mathrm{He}-\mathrm{B}$ the vortices which terminate in the bulk superfluid due to the complete transfer of the angular momenta of paired ${ }^{3} \mathrm{He}$ atoms, which constitute such vortices, to the orbital angular momenta and spins of the atoms may be produced. Examples of such vortices in superfluid ${ }^{3} \mathrm{He}-\mathrm{B}$ are vortices of the "hedgehog” type.

7. In vortex cores of superfluid ${ }^{3} \mathrm{He}-\mathrm{B}$, the precession of spins of ${ }^{3} \mathrm{He}$ atoms takes place. This follows from the experiments on magnetic resonance conducted with rotating superfluid ${ }^{3} \mathrm{He}-\mathrm{B}$.

8. The Einstein-de Haas effect (or the Richardson effect) takes place: in the case of ${ }^{3} \mathrm{He}-\mathrm{B}$ this is a rotation of a volume of superfluid ${ }^{3} \mathrm{He}-\mathrm{B}$ caused by magnetization of the superfluid. Since the magnetization of ${ }^{3} \mathrm{He}$ atoms means as well their spin polarization, then the Einstein-de Haas effect is in fact the rotation of a superfluid volume provided that $\partial \mathbf{S}_{l} / \partial t \neq 0$, where $\mathbf{S}_{l}$ is the total spin of the volume under consideration.

9. The vortex core in superfluid ${ }^{3} \mathrm{He}-\mathrm{B}$ is an electric dipole whose electric dipole moment may be aligned with the vortex axis.

10. In the vortex core of superfluid ${ }^{3} \mathrm{He}-\mathrm{B}$, due to unidirectional orientation of spins of ${ }^{3} \mathrm{He}$ atoms the phase transition occurs (the superfluid A phase or spontaneously ferromagnetic $\beta$-phase may be produced). The phase transition leads to a change in the inertial properties of the superfluid in the vortex core in contrast with the inertial properties of the superfluid in the rest of the volume.

11. The mutually relative motion of particles constituting a Cooper pair in superfluid ${ }^{3} \mathrm{He}-\mathrm{B}$ corresponds to the $p$-wave state. In this state, attractive forces act between like-charged particles with spins aligned in the same direction.

12. In superfluid ${ }^{3} \mathrm{He}-\mathrm{B}$, there may exist regions with identically oriented and coherently precessing spins of ${ }^{3} \mathrm{He}$ atoms, such regions being referred to as homogeneously precessing domains.

13. In superfluid ${ }^{3} \mathrm{He}-\mathrm{B}$ the nutation and precession angles of precessing spins of atoms are angles of orientation of the order parameter, and there are processes that tend to equalize both the values of precession angles and the values of nutation angles throughout the liquid volume. Such processes in superfluid ${ }^{3} \mathrm{He}-\mathrm{B}$ are spin supercurrents. Let us consider two homogeneously precessing domains $\left(H P D_{1}\right.$ and $\left.H P D_{2}\right)$ having respective precession frequencies $\boldsymbol{\omega}_{1}$ and $\boldsymbol{\omega}_{2}$, precession angles $\alpha_{1}$ and $\alpha_{2}$, nutation angles $\beta_{1}$ and $\beta_{2}$ and the same values of spins $S$. Frequencies $\boldsymbol{\omega}_{1}$ and $\boldsymbol{\omega}_{2}$ are aligned with axis $\mathbf{z}$ (see Figure 3).

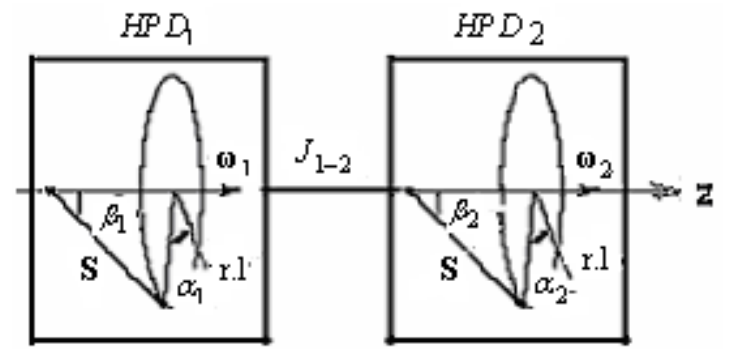

Figure 3. Spin structures $H P D_{1}$ and $H P D_{2}: \boldsymbol{\omega}_{1}$ and $\boldsymbol{\omega}_{2}$ are precession frequencies, $\alpha_{1}$ and $\alpha_{2}$ are precession angles, $\beta_{1}$ and $\beta_{2}$ are nutation angles, $\mathbf{S}$ is spin; r.l. is the reference line, $J_{1-2}$ is spin supercurrent

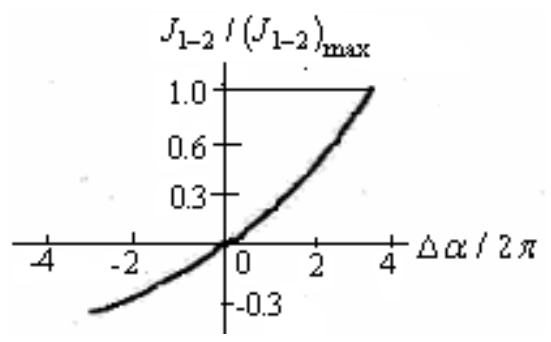

Figure 4. Normalized spin supercurrent $J_{1-2} /\left(J_{1-2}\right)_{\max }$ as a function of precession phase difference $\Delta \alpha /(2 \pi)$ 
At $\beta_{1}=\beta_{2}$ the experimentally obtained dependency of spin supercurrent $J_{1-2}$ between the two of homogeneously precessing domains on the difference $\alpha_{1}-\alpha_{2}$ is shown in Figure 4.

Within a definite accuracy the experimentally obtained dependency can be approximated by the linear function:

$$
J_{1-2}=k\left(\alpha_{1}-\alpha_{2}\right)
$$

where $k$ is a proportionality factor. Theoretically, the spin supercurrent is dissipation free and does not depend on the distance.

\section{The Vortex-Wave Process in the Superfluid Physical Vacuum}

\subsection{The Equations of Vortex-Wave Process}

As the properties of the SPV are like those of superfluid ${ }^{3} \mathrm{He}-\mathrm{B}$, then according to properties $1-5$ and 8 (Section 2) the dynamics of the SPV in the nonstationary case in a vorticity region can be described by a model of an ideal spin liquid, i.e. the liquid with intrinsic degrees of freedom and without shear (linear) viscosity. The intrinsic degrees of freedom are characterized by both vector $\mathbf{A}$ of total spin of infinitesimal volume element of the liquid and the rotational viscosity determining transformation of macrorotations in the medium into microrotations.

The equation for the motion of liquid, if we neglect the momentum flux through the boundary, will be [23]:

$$
\rho \frac{\partial \mathbf{u}}{\partial t}=\left(\nabla_{i} P_{j i}\right) \exists_{j}
$$

where $\rho$ is the liquid density, $\mathbf{u}$ is the velocity of the moving liquid, $\exists_{j}$ is the basis vector, and $P_{j i}$ are components of the stress tensor in this basis vector $(i, j=1$, $2,3, \ldots)$. In the Cartesian coordinate system

$$
\nabla_{i} P_{j i}=\frac{\partial P_{j 1}}{\partial x}+\frac{\partial P_{j 2}}{\partial y}+\frac{\partial P_{j 3}}{\partial z}
$$

Using the formalism developed in mechanics for writing equations of motion of continuum with intrinsic degrees of freedom, we shall express the stress tensor components $P_{j i}$ as follows [23]:

$$
P_{j i}=B_{j i l m} A_{l m}, l, m=1,2,3, \ldots
$$

where $A_{I m}$ are the components of the antisymmetric tensor of intrinsic rotations. In the SPV model the tensor characterizes the total spin of infinitesimal volume element of the liquid. We define $A_{l m}$ as:

$$
A_{l m}=\sum_{q=1}^{3} \varepsilon_{q l m} a_{q}=a_{1} \varepsilon_{1 l m}+a_{2} \varepsilon_{2 l m}+a_{3} \varepsilon_{3 l m}
$$

The coefficients $\quad \varepsilon_{q l m}$ are defined as follows:

$$
\varepsilon_{q l m}=\left\{\begin{array}{l}
1 \text { at } q, l, m=1,2,3 ; 2,3,1 ; 3,1,2 \\
-1 \text { at } q, l, m=2,1,3 ; 1,3,2 ; 3,2,1 \\
0 \text { in other cases. }
\end{array}\right.
$$

$\varepsilon_{q l m}$ can be expressed through Kronecker's delta $\delta$, then equation (6) will take the form:

$$
\begin{aligned}
& A_{l m}=a_{1}\left(\delta_{l 2} \delta_{m 3}-\delta_{l 3} \delta_{m 2}\right)+a_{2}\left(\delta_{l 3} \delta_{m 1}-\delta_{l 1} \delta_{m 3}\right) \\
& +a_{3}\left(\delta_{l 1} \delta_{m 2}-\delta_{l 2} \delta_{m 1}\right)
\end{aligned}
$$

The coefficients $B_{j i l m}$ depend on the properties of the liquid; thus they must form a rank IV tensor, and they must not vary with any orthogonal transformation of coordinates. As it was mentioned above, the medium modeling the properties of the SPV is characterized by intrinsic degrees of freedom and rotational viscosity, which manifests itself in nonstationary cases; there being no shear viscosity there. For such a medium, in the linear case, the tensor in question, provided the space is homogeneous and isotropic (property 1, Section 2), has the following components in the Cartesian coordinate system $[17,24]$ :

$$
B_{j i l m}=b_{1} \delta_{j m} \delta_{i l}+b_{2} \delta_{j i} \delta_{l m}+b_{3} \delta_{j l} \delta_{i m}
$$

where $b_{1}, b_{2}, b_{3}$ are constants. Substituting the righthand members of equations (7) and (8) in equation (5), we obtain the following expression for the stress tensor components:

$$
\begin{aligned}
& P_{j i}=\left(b_{1} \delta_{j m} \delta_{i l}+b_{2} \delta_{j i} \delta_{l m}+b_{3} \delta_{j l} \delta_{i m}\right) \\
& \cdot\left[a_{1}\left(\delta_{l 2} \delta_{m 3}-\delta_{l 3} \delta_{m 2}\right)\right. \\
& \left.+a_{2}\left(\delta_{l 3} \delta_{m 1}-\delta_{l 1} \delta_{m 3}\right)+a_{3}\left(\delta_{l 1} \delta_{m 2}-\delta_{l 2} \delta_{m 1}\right)\right] \\
& =b_{1}\left[a_{1}\left(\delta_{j 3} \delta_{i 2}-\delta_{j 2} \delta_{i 3}\right)+a_{2}\left(\delta_{j 1} \delta_{i 3}-\delta_{j 3} \delta_{i 1}\right)\right. \\
& \left.+a_{3}\left(\delta_{j 2} \delta_{i 1}-\delta_{j 1} \delta_{i 2}\right)\right]+b_{3}\left[a_{1}\left(\delta_{j 2} \delta_{i 3}-\delta_{j 3} \delta_{i 2}\right)\right. \\
& \left.+a_{2}\left(\delta_{j 3} \delta_{i 1}-\delta_{j 1} \delta_{i 3}\right)+a_{3}\left(\delta_{j 1} \delta_{i 2}-\delta_{j 2} \delta_{i 1}\right)\right] .
\end{aligned}
$$

Taking into account (6) in (9) we have:

$$
\begin{aligned}
& P_{j i}=b_{1}\left(-a_{1} \varepsilon_{1 j i}-a_{2} \varepsilon_{2 j i}-a_{3} \varepsilon_{3 j i}\right) \\
& +b_{3}\left(a_{1} \varepsilon_{1 j i}+a_{2} \varepsilon_{2 j i}+a_{3} \varepsilon_{3 j i}\right) \\
& =-\left(b_{1}-b_{3}\right) A_{j i} .
\end{aligned}
$$

Further we shall rewrite equation (3), using (4) and (10):

$$
\begin{aligned}
& -\frac{\rho}{b_{1}-b_{3}} \frac{\partial \mathbf{u}}{\partial t}=\left(\frac{\partial A_{11}}{\partial x}+\frac{\partial A_{12}}{\partial y}+\frac{\partial A_{13}}{\partial z}\right) \exists_{1} \\
& +\left(\frac{\partial A_{21}}{\partial x}+\frac{\partial A_{22}}{\partial y}+\frac{\partial A_{23}}{\partial z}\right) \exists_{2} \\
& +\left(\frac{\partial A_{31}}{\partial x}+\frac{\partial A_{32}}{\partial y}+\frac{\partial A_{33}}{\partial z}\right) \exists_{3} \\
& =\left(\frac{\partial a_{3}}{\partial y}-\frac{\partial a_{2}}{\partial z}\right) \exists_{1}+\left(\frac{\partial a_{1}}{\partial z}-\frac{\partial a_{3}}{\partial x}\right) \exists_{2} \\
& +\left(\frac{\partial a_{2}}{\partial x}-\frac{\partial a_{1}}{\partial y}\right) \exists_{3}=\text { curlA },
\end{aligned}
$$

or

$$
\frac{\partial \mathbf{u}}{\partial t}=\frac{b_{3}-b_{1}}{\rho} \text { curlA } .
$$

According to property 8 (Section 2), the Einstein - de Haas effect takes place in the SPV and the following can be written: 


$$
\frac{\partial \mathbf{A}}{\partial t}=-\chi \operatorname{curl} \mathbf{u}
$$

where $\chi$ is the proportionality coefficient

$$
\chi>0
$$

Let us introduce the constant $\xi$ :

$$
\xi=\sqrt{\frac{\chi\left(b_{3}-b_{1}\right)}{\rho}}
$$

According to (14) and taking into account (13), the necessary condition for the quantity $\xi$ to have a physical meaning is the following: $b_{3}-b_{1}>0$.

Using equations (14) in (11) and (12) we obtain:

$$
\begin{aligned}
& \frac{\partial \mathbf{u}}{\partial t}=\xi \cdot \operatorname{curl}\left(\frac{\xi}{\chi} \mathbf{A}\right) \\
& \frac{\partial\left(\frac{\xi}{\chi} \mathbf{A}\right)}{\partial t}=-\xi \cdot \operatorname{curl} \mathbf{u}
\end{aligned}
$$

These equations describe a spin-wave process propagating at a speed $\xi$ in the SPV. According to property 5 (Section 2) spin arises in vortices, thus the process described by equations (15) and (16) is a vortexwave process.

In the conventional, i.e. not superfluid, medium the intrinsic rotations cannot propagate as a wave process because the diffusion of the vortices is significant. However, under property 4 (Section 2) quantized vortices do not diffuse in the SPV. Hence if in the SPV there is a mechanism that suppresses a vortex in a point of space and simultaneously transports the vortex energy to adjacent points, then the vortex process will propagate through the SPV.

Let us denote:

$$
\mathbf{Y}=-\frac{\xi}{\chi} \mathbf{A}
$$

Let us consider the physical sense of variable $\mathbf{Y}$, which according to (13), (14) and (17) is directed oppositely to A, is proportional to the magnitude $A$ and has the dimension of velocity. Let us address the property 11 of superfluid ${ }^{3} \mathrm{He}-\mathrm{B}$ (Section 2). Under this property, in a vortex core produced in the SPV the motion of electrically unlike particles occurs in opposite directions, causing the electric polarization of the vortex, thus the vortex can be regarded as an electric dipole (property 9, Section 2). Consequently, the variable $\mathbf{Y}$ may denote the speed of this motion relative to the SPV:

Using (17) in (15) and (16), we obtain:

$$
\begin{aligned}
\frac{\partial \mathbf{u}}{\partial t} & =\xi \cdot \operatorname{curl} \mathbf{Y} \\
\frac{\partial \mathbf{Y}}{\partial t} & =-\xi \cdot \text { curl } \mathbf{u}
\end{aligned}
$$

According to (18) and (19), in the vortex-wave process discussed there is a transfer of energy associated with the motion of the SPV outside the vortex (velocity $\mathbf{u}$ ) to the energy associated with the motion of the SPV in the vortex core (velocity $\mathbf{Y}$ ).

\subsection{The Components of the Energy of the Vortex-Wave Process in the SPV}

\subsubsection{The specific kinetic energy of the SPV, associated with the speed $\mathbf{Y}$}

The specific kinetic energy of the SPV, $W_{Y}$, is associated with the speed $Y$ of motion of electrically charged particles that constitute the vortex core: $W_{y}=\left(\rho_{+}+\rho_{-}\right) \cdot Y^{2} / 2 .\left(\rho_{+}\right.$and $\rho_{-}$are the density of positively and negatively charged particles in the medium respectively). Taking into account that $\rho_{1}+\rho_{2}=\rho$, we obtain:

$$
W_{Y}=\rho Y^{2} / 2
$$

The mutually relative motion of unlike-charged particles in the vortex core results in electric polarization of the SPV in the vortex core. The specific energy $W_{E}$ of the produced electric field $\mathbf{E}$ [25] is determined as:

$$
W_{E}=E^{2} / 8 \pi
$$

Assuming that all the energy $W_{Y}$ is converted into the energy $W_{E}$ of the produced electric field $\mathbf{E}$, assuming that E $\uparrow \downarrow \mathbf{A}$ and using Eqs. (20) and (21) we have:

$$
\mathbf{E}=2 \sqrt{\pi \rho} \cdot \mathbf{Y}
$$

Using (22) in (18) and (19) we obtain:

$$
\begin{aligned}
& \frac{\partial(\sqrt{4 \pi \rho} \cdot \mathbf{u})}{\partial t}=-\xi \cdot \operatorname{curl} \mathbf{E} \\
& \frac{\partial \mathbf{E}}{\partial t}=\xi \cdot \operatorname{curl}(\sqrt{4 \pi \rho} \cdot \mathbf{u})
\end{aligned}
$$

Equations (23) and (24) describe the process of propagation of the electric polarization of vortex in the vortex-wave process in the SPV at speed $\xi$. It is noteworthy that curlE in equation (23) characterizes not a vortex motion in an electric field, but a circulation of $\mathbf{E}$, which circulation is a result of electric polarization of the SPV in the vortex cores.

\subsubsection{The specific kinetic energy of the SPV, associated with the velocity $u$}

The specific kinetic energy of the SPV, $W_{u}$, associated with the speed of motion of the SPV, $u$, is:

$$
W_{u}=\rho u^{2} / 2
$$

Analyzing Eqs. (23) and (24), we shall introduce the following denotation:

$$
\mathbf{X}=\sqrt{4 \pi \rho} \cdot \mathbf{u}
$$

Using (25) in (26), we have:

$$
W_{u}=X^{2} /(8 \pi)
$$

Using (26) in (23) and (24), we obtain: 


$$
\begin{gathered}
\frac{\partial \mathbf{X}}{\partial t}=-\xi \cdot \operatorname{curl} \mathbf{E} \\
\frac{\partial \mathbf{E}}{\partial t}=\xi \cdot \operatorname{curl} \mathbf{X}
\end{gathered}
$$

Let us discuss the character of dependence of pressure $p$ in the SPV on speed $u$. Hydrodynamically, the stationary motion of a superfluid is described [26] by the following equation: $\rho u^{2} / 2+\rho \mu=$ const, where $\mu$ is the chemical potential, $\rho$ is the density of the superfluid. The potential $\mu$ may not reveal itself inside the vortex because of a phase transition inside the vortex core (property 10, Section 2). In this connection, the action of the superfluid on the vortex core can be described mathematically by introducing pressure $p$ on the boundary of the vortex core in the SPV [24]. The sign of the pressure depends on the nature of internal stresses in the medium. If the internal stresses are like "omniradial tensions" [23], the pressure will be negative. Since a "break" of particles constituting the vortices into electrically unlike parts takes place in the vortices of the SPV, it is possible to assume that in the vorticity region of the SPV the internal stresses are like "omniradial tensions", thus it may be assumed:

$$
\rho u^{2} / 2-p=\text { const }
$$

That is, the pressure in the vorticity region may be negative.

In favor of validity of Eq. (30) there are results of experiments with rotating superfluid ${ }^{3} \mathrm{He}-\mathrm{B}$ : the so called vortex molecules consisting of two vortices with angular momenta oriented in parallel [19] have been detected. This is characteristic of the medium with negative pressure.

\subsubsection{The energy associated with the precessing vortex spin}

According to property 7 (Section 2), the precession of spins of particles that constitute the vortex cores of the SPV takes place. The frequency of precession, $\omega_{v}$, determines the energy associated with precessing spins of microparticles, $W_{s}^{v}[23]$ :

$$
W_{s}^{v}=S_{v} \omega_{v}
$$

where $S_{v}$ is the total value of precessing spins.

Since, under property 5 (Section 2), in the SPV there is a transfer of angular momentum of the pairs of particles that constitute the vortex to the spins of the particles, then, according to property 3 (Section 2), the total spin $S_{v}$ of the particles in the vortex may be equal to $n \hbar, n=1,2$, $3, \ldots$ If $n=1$, we have:

$$
S_{v}=\hbar
$$

Then equation (31) will be expressed as:

$$
W_{s}^{v}=\hbar \omega_{v}
$$

3.2.4. The energy associated with the inertial properties of vortex
Properties 5, 7, 10 and 11 from Section 2 (the spin orientation, spin precession and nonzero forces between the particles that constitute the vortex, the forces being aligned with the spin of the particles) allow one to introduce the mass of vortex, $m_{v}$ and also the motion of the mass inside the vortex (Figure 5).

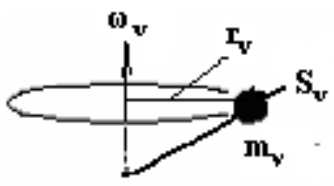

Figure 5. Diagram of precession motion of mass $m_{v}$ inside a vortex. $\omega_{v}$ is the vortex precession frequency, $S_{v}$ is the total vortex spin; $r_{v}$ is the radius of the circular motion of mass $m_{v}$ inside the vortex

Thus the energy of the vortex as an entity with mass can be represented by two terms: the kinetic energy $W_{m_{v}}^{k}=m_{v} \xi^{2} / 2$ of the center of mass, in which all the vortex mass is assumed to be contained, and the energy $W_{m_{v}}^{S}$ associated with the precession of vortex spin. The energy $W_{m_{v}}^{S}$ is defined as [23]: $W_{m_{v}}^{S}=J_{m_{v}} \omega_{v}^{2} / 2$, where $J_{m_{v}}$ is the moment of inertia associated with mass $m_{v}$. The quantity $J_{m_{v}} \omega_{\mathcal{M}}$ determines the angular momentum of mass $m_{v}$ and it must be equal to the angular momentum of vortex, $S_{v}$, or according to (23):

$$
J_{m_{v}} \omega_{v}=\hbar \text {. }
$$

Thus the total energy $W_{m_{v}}$ of mass $m_{v}$ moving relative to the SPV is determined as:

$$
W_{m_{v}}=W_{m_{v}}^{k}+W_{m_{v}}^{v}=\frac{m_{v} \xi^{2}}{2}+\frac{\hbar \omega_{v}}{2}
$$

Being a characteristic of the same entity, i.e. the vortex, the energy $W_{m_{v}}$ associated with mass must be equal to the energy of the vortex, contained in its spin system, $W_{s} ; W_{s}$ being the sum of energy $W_{s}^{v}$ (see Eq. (31)) and energy associated with the motion of spin in space at speed $\xi$. Denote the latter energy as $W_{s}^{k}$, thus:

$$
W_{s}=W_{s}^{v}+W_{s}^{k}
$$

If the motion of spin in space is inertia free, kinetic energy cannot be associated with the motion, that is:

$$
W_{s}^{k}=0
$$

Then, using Eqs. (35) and (36), we obtain the following expression for the vortex mass:

$$
m_{v}=\hbar \omega_{v} / \xi^{2}
$$

Because of creation of mass in vortices, the wavevortex process in the physical vacuum having the properties of superfluid ${ }^{3} \mathrm{He}-\mathrm{B}$ is at the same time a process in a gravitational field. Therefore, the speed of wave-vortex process will be determined by the properties of gravitational field and will tend to become equal to a 
definite value in the vicinity of the sources of the field, relative to the sources (i.e. the entities possessing a mass).

Note. In the simplest case of circular motion (with radius $r_{v}$, see Figure 5) of mass $m_{v}$ inside the vortex the quantity $J_{m_{v}}$ is determined as $J_{m_{v}}=m_{v} r_{v}^{2}$. Using this equation in Eqs. (34) and (38), we obtain the following expression:

$$
2 \pi r_{v}=2 \pi \xi / \omega_{v}
$$

That is, the length of the circle where the mass moves is equal to the wavelength of the vortex-wave process that is described by equations (15) and (16) (or (18)-(19), (23)(24), (28)-(29)) and propagates at speed $\xi$.

\section{The Interaction of Vortices in the SPV}

According to properties 5, 7 and 12 of superfluid ${ }^{3} \mathrm{He}-\mathrm{B}$, the vortices in this fluid can be considered to be homogeneously precessing domains; thus according to property 13 (Section 2) there may exist a process between the vortices that makes equal the respective angles of precession and angles of nutation of spins of particles that constitute the vortices. This process is spin supercurrent. Let us discuss the features of variation of characteristics of vortices using as an example the interaction of $H P D_{1}$ and $H P D_{2}$ shown in Figure 3. The precession angles $\alpha_{1}$ and $\alpha_{2}$ are related to the precession frequencies $\omega_{1}$ and $\omega_{2}$ (in the special case of independence of precession frequencies on time $t$ ), by equations:

$$
\begin{aligned}
& \alpha_{1}=\omega_{1} t+\alpha_{1}^{0} \\
& \alpha_{2}=\omega_{2} t+\alpha_{2}^{0}
\end{aligned}
$$

where $\alpha_{1}^{0}$ and $\alpha_{2}^{0}$ are respectively the values of the precession angles for $H P D_{1}$ and $H P D_{2}$ at $t=0$. If spin supercurrent $J_{1-2}$ causes changes in precession angles $\alpha_{1}$ and $\alpha_{2}$ (these changes are denoted as $\Delta \alpha_{1}$ and $\Delta \alpha_{2}$ ), then according to Eqs. (40) and (41) this results in changes in frequencies $\boldsymbol{\omega}_{1}$ and $\boldsymbol{\omega}_{2}$ (the changes are denoted as $\Delta \omega_{1}$ and $\left.\Delta \omega_{2}\right)$. Under Eq. (31), a change in the $H P D_{1}$ energy, $\left(\Delta W_{s}^{v}\right)_{1}$, and a change in the $H P D_{2}$ energy, $\left(\Delta W_{s}^{v}\right)_{2}$, are determined as:

$$
\begin{aligned}
& \left(\Delta W_{s}^{v}\right)_{1}=S \cdot \Delta \omega_{1} \\
& \left(\Delta W_{s}^{v}\right)_{2}=S \cdot \Delta \omega_{2}
\end{aligned}
$$

Since energy conservation in superfluid ${ }^{3} \mathrm{He}-\mathrm{B}$ is valid and the spin supercurrent is dissipation free, the equalization of respective characteristics of $H P D_{1}$ and $H P D_{2}$ results in:

$$
\left(\Delta W_{s}^{v}\right)_{1}=-\left(\Delta W_{s}^{v}\right)_{2}
$$

Then according to Eqs. (42) and (43):

$$
\Delta \omega_{1}=-\Delta \omega_{2}
$$

According to Eqs. (40), (41) and (45):

$$
\Delta \alpha_{1}=-\Delta \alpha_{2}
$$

The spin supercurrent in superfluid ${ }^{3} \mathrm{He}-\mathrm{B}$ does not depend on the distance between homogeneously precessing domains. Interaction between homogeneously precessing domains is most effective, that is, the respective precession and nutation angles of the domains tend to become equal, provided the difference between their precession frequencies, $\Delta \omega$, satisfies the following condition:

$$
\Delta \omega \rightarrow 0
$$

\section{Comparison of Properties of Photons and Properties of Vortex-Wave Process in the SPV}

Let us compare the properties of photon discussed in Introduction and the properties of vortex-wave process discussed in Sections 3 and 4, which propagates in the SPV.

\section{C-photon.}

1. The representation of photon by a classical wave packet described by Maxwell's equations is in agreement with the conclusions of Section 3: a vortex-wave process which is described by the equations of Maxwell's type may propagate in the SPV (see Eqs. 15-16, 18-19, 23-24, 28-29).

2. The electric properties of photon are similar to those of vortices in the vortex-wave process in the SPV (see Eqs. 23 and 24).

3. The preservation of the plane of electric component of photon is similar to the preservation of the plane of electric component of vortex in the vortex-wave process in the SPV. The vortices of the vortex-wave process are characterized by the precession of spins of the particles that constitute the vortex; this precession determines the gyroscopic properties of the vortex-wave process.

4. The equalization of the light speed in any inertial frame of reference to the absolute constant $c$ is similar to the analogous property of vortex-wave process in the SPV: due to creation of mass in the vortex, the vortex-wave process is also a process in a gravitational field. Therefore, the speed of the vortex-wave process is determined by the properties of gravitational field and may tend to become equal to a definite value in the vicinity of the sources of the field, relative to the sources (i.e. the entities possessing a mass).

5. The photon has a magnetic component.

Equations (28) and (29) describing the vortex-wave process imply that variable $\mathrm{X}$ may be a candidate for the role of magnetic component. According to Eqs. (25)-(27), variable $X$ is associated with the velocity $\boldsymbol{v}$ of motion of the SPV in the vorticity region. It is shown in [23] that there is a complete analogy between the structures of formulas describing the magnetic interactions of currentcarrying wires and the structures of formulas describing the interaction of vortices in an ideal incompressible liquid having negative pressure. (Note that according to (30) the SPV in the vorticity region is such a medium.) In addition, 
the field's magnetic induction $\mathrm{B}$ is related to the velocity $\mathrm{u}$ of the liquid of density $\rho_{l}$ by equation $\mathbf{B}=\sqrt{4 \pi \rho_{l}} \cdot \mathbf{u}$ that is similar to equation (26) which relates variable $\mathrm{X}$ to the velocity of the SPV, $\boldsymbol{v}$.

M-photon. The characteristics of M-photon's mass ("kinetic" mass) $m_{p h}$, defined as $m_{p h}=\hbar \omega_{p h} / c^{2}$, angular nomentum $\hbar$, energy $\hbar \omega_{p h}$ are similar, according to (38), (32) and (33), to the respective characteristics of vortices in the vortex-wave process: $m_{v}=\hbar \omega_{v} / \xi^{2}, S_{v}=\hbar, W_{s}^{v}=\hbar \omega_{v} \quad\left(\omega_{v}\right.$ is the precession frequency of vortex spin, $\xi$ is the speed of the vortexwave process).

Note. The energy of M-photon is not equal to the kinetic energy of photon's mass, $\hbar \omega_{p h} / 2$, which suggests the existence of intrinsic motions of the mass in a photon. In [21] the expression for the energy of photon's mass, $W_{m_{p h}}$, was derived:

$$
W_{m_{p h}}=\frac{m_{p h} w^{2}}{2}+\frac{\hbar \omega_{p h}}{2}
$$

where $m_{p h}$ is the speed of photon with respect to the reference frame. It is shown in Appendix that on the basis of Eq. (47) and also the Galilean addition of velocities it is possible to derive equations for the description of the transverse and longitudinal Doppler effects, which coincide to a high accuracy with similar equations derived in special relativity [27].

The expression (47) is similar to expression (35) that determines the energy associated with inertial properties of a vortex in the vortex-wave process, the SPV.

Q-photon. The properties of quantum correlations discussed in Introduction are similar to the properties (discussed in Section 4) of interaction of vortices in superfluid ${ }^{3} \mathrm{He}-\mathrm{B}$ through spin supercurrents.

1. The independence of quantum correlations of the distance between the correlated photons is similar to the analogous property of spin supercurrent in superfluid ${ }^{3} \mathrm{He}-$ $\mathrm{B}$ : the independence of the distance between the spin structures, between which it arises.

2. That quantum correlations between photons of the same frequency, emitted by different sources, may take place does not contradict the condition of effective interaction of homogeneously precessing domains in the SPV. According to Eq. (46), the efficacy of spin supercurrents is maximum if the interacting homogeneously precessing domains have equal precession frequencies.

3. That quantum correlations of photons may take place not only in the process of simultaneous detection of both photons is in agreement with the property of interaction of homogeneously precessing domains through spin supercurrents in the SPV. Spin supercurrents arise between such domains under the only condition that there are nonzero gradients of nutation or/and precession angles between them.

4. The relation between the changes in phases of the light waves in the two-photon interference (Eq. (1)), which is a manifestation of quantum nonlocality, is similar, according to Eqs. (43)-(45), to the features of variation of energy, frequency and precession angle in interaction of vortices in the SPV through spin supercurrents.

5. The quantum correlations may be due to "superluminal" forces between the correlated photons. This property of quantum correlations is similar to the property of spin supercurrent arising between homogeneously precessing domains in the SPV. Indeed, the spin supercurrent can be considered a transfer of spin projection gradient onto a preferential direction and, according to Eqs. (36) and (37), can be considered an inertia free process. Therefore, spin supercurrent is not accompanied by the birth of a mass and consequently is not a process in the gravitational field. Thus the speed of spin supercurrent in the SPV may have any value with respect to singularities in the gravitational field, and therefore may be greater than the speed of vortex-wave process in the SPV, which is accompanied by the birth of a mass in vortices (see Eq. (38)) [28,29].

\section{Conclusion}

Endowing the physical vacuum with the properties of superfluid ${ }^{3} \mathrm{He}-\mathrm{B}$ allows one to represent the main properties of light, i.e. electric, magnetic, the spin, "kinetic mass", quantum correlation of the photons, the constancy of the speed of light in any inertial frame of reference as properties of a vortex-wave process in the medium similar to superfluid ${ }^{3} \mathrm{He}-\mathrm{B}$.

\section{Appendix. The Doppler Effect for Light}

Consider an inertial frame of reference linked to the detector, where the source of light of mass $M$ is moving at velocity $\mathbf{v}_{0}$. The source of light is assumed to be at rest with respect to the Earth, the speed of light relative to the source is equal to $c$. We shall derive the formulas describing the longitudinal and transverse Doppler effects on the basis of the Galilean addition of velocities.

It is experimentally established (for example, in the photoelectric effect) that the absorption of light occurs in quanta of energy $\hbar \omega_{d}$, where $\omega_{d}$ is the frequency of the light detected. If the mass of the detector as well as the mass of the source are great, the motion of the detector due to the pressure of light is negligible and thus the energy loss for the photon may be neglected too. Then in the interaction of the photon and the detector all the energy $U_{p h}$ of the photon in the inertial frame is equal to the detected energy $\hbar \omega_{d}$. Let us apply Eq. (47) to the photon energy. Provided $\xi=c$ we have:

$$
\hbar \omega_{d}=U_{p h}=\frac{\hbar \omega_{0}\left(\mathbf{c}+\mathbf{v}_{0}\right)^{2}}{2 c^{2}}+\frac{\hbar \omega_{0}}{2}
$$

where $\omega_{0}$ is the photon frequency in the frame of the source of photons. Let us introduce the vector $\mathbf{w}$ directed from the source to the detector (Figure 6):

$$
w=c+v_{0}
$$




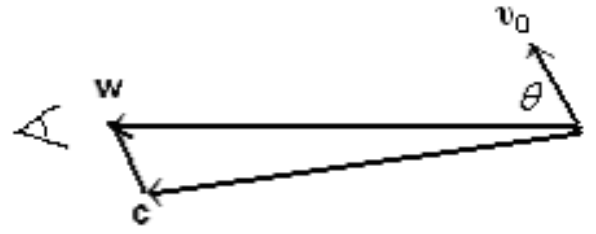

Figure 6. Ray path diagram: $\mathbf{c}$ is the light velocity relative to the source, $\mathbf{v}_{0}$ is the source velocity relative to the detector, $\mathbf{w}$ is the resulting light velocity directed from the source to detector, $\theta$ is the angle between $\mathbf{w}$ and $\boldsymbol{v}_{0}$

Introducing $\theta$, the angle between vectors $\mathbf{w}$ and $\mathbf{v}_{0}$, denoting $\beta=w / c$ and using (A.2), the equation (A.1) can be expressed as:

$$
\begin{aligned}
& \hbar \omega_{d}=\hbar \omega_{0}\left(1+\frac{\left(\mathbf{v}_{0} \mathbf{w}\right)}{c^{2}}-\frac{v_{0}^{2}}{2 c^{2}}\right) \\
& =\hbar \omega_{0}\left(1+\frac{w}{c} \beta \cos \theta-\frac{1}{2} \beta^{2}\right)
\end{aligned}
$$

The quantity $w / c$ can be derived from the following equation: $\quad c^{2}=\left(\mathbf{w}-\mathbf{v}_{0}\right)^{2}=w^{2}+v_{0}^{2}-2 w \cdot v_{0} \cos \theta$. Dividing both sides of this equation by $c^{2}$, we obtain $(w / c)^{2}-2 \beta \cos \theta(w / c)-\left(1-\beta^{2}\right)=0$. Taking into account that $w / c>0$ and assuming that $\beta<<1$ we obtain only one solution:

$$
w / c=\beta \cos \theta+\sqrt{1-\beta^{2} \sin ^{2} \theta}
$$

Substituting the right side of (A.4) in (A.3), we have:

$\omega_{d}=\omega_{0}\left(1+\beta \cos \theta\left(\beta \cos \theta+\sqrt{1-\beta^{2} \sin ^{2} \theta}\right)-\frac{\beta^{2}}{2}\right)(\mathrm{A} .5)$

To an accuracy of $\beta^{3}$ the equation (A.5) may be written as:

$$
\begin{aligned}
& \omega_{d}=\omega_{0}\left(1+\beta \cos \theta+\beta^{2} \cos ^{2} \theta-\frac{\beta^{2}}{2}\right. \\
& \left.-\frac{\beta^{3}}{2} \cos \theta+\frac{\beta^{3}}{2} \cos ^{3} \theta+o\left(\beta^{3}\right)\right)
\end{aligned}
$$

where $o\left(\beta^{3}\right)$ are summands of a lower order of magnitude than $\beta^{3}$. Equation (A.6) coincides accurate to $\beta^{2}$ inclusive (at $\theta=\pi / 2$ accurate to $\beta^{3}$ inclusive) with experimentally tested equation describing Doppler's effect in special relativity [2]:

$$
\begin{aligned}
& \omega_{d}=\omega_{0} \frac{\sqrt{1-\beta^{2}}}{1-\beta \cos \theta} \\
& =\omega_{0}\left(1+\beta \cos \theta+\beta^{2} \cos ^{2} \theta\right. \\
& \left.-\frac{\beta^{2}}{2}-\frac{\beta^{3}}{2} \cos \theta+\beta^{3} \cos ^{3} \theta+o\left(\beta^{3}\right)\right)
\end{aligned}
$$

Let us consider the special cases. If $\cos \theta=0$, the formula describing the transverse Doppler effect follows from (A.6): $\omega_{d}=\omega_{0}\left(1-\beta^{2} / 2\right)$. If $\cos \theta=1$ or $\cos \theta=-1$, the formula describing the longitudinal Doppler effect follows from (A.6): $\omega_{d}=\omega_{0}\left(1+\beta+\beta^{2} / 2\right)$ or $\omega_{d}=\omega_{0}\left(1-\beta+\beta^{2} / 2\right)$.

Thus using the representation of the photon energy by equation (A.1), we obtain the equation (A.5) describing all special cases of the Doppler effect.

\section{Statement of Competing Interests}

The author has no competing interests.

\section{List of Abbreviations}

SPV - Superfluid Physical Vacuum

\section{References}

[1] Klyshko D.N, "Quantum optics: quantum, classical, and metaphysical aspect," Physics Uspekhi, 37, 1097-1122, 1994.

[2] Einstein A, Fundamental Ideas and Methods of the Theory of Relativity, 1920.

[3] Alvager T., Barley J.M, "Test of the second postulate of Special Relativity in the GeV region," Physical Letters, 12, 260, 1964.

[4] Compton A.N, "The Spectrum of Scattered X-Rays," Physical Review, 22, 409, 1923.

[5] Tittel W., Brendel J., Gisin B., Herzog T., Zbinden H., Gisin N, "Experimental demonstration of quantum-correlations over more than 10 kilometers," Physical Review A, 57, 3229, 1998.

[6] Belinskii A.V, "Quantum nonlocality and the absence of a priori values for measurable quantities in experiments with photons," Physics Uspekhi, 46, 877-881, 2003.

[7] Hanbury Brown, R., Twiss, R.Q, "A new type of interferometer for use in radio astronomy,” Philosophical. Magazine, 45, 663-682, 1954.

[8] Sinha K.P., Sivaram C., Sudarshan E.C.G, "The Superfluid Vacuum State. Time-Varing Cosmological Constant, and Nonsingular Cosmological Models," Foundations of Physics, 6, No. 6, 717-726, 1976.

[9] Bauerle C., Bunkov Yu.M., Fisher S.N., Godfrin H., Pickett G.R, "Laboratory simulation of cosmic string formation in the early Universe using superfluid ${ }^{3} \mathrm{He}$," Nature, 382, 332, 1996.

[10] Volovic, G.E, The Universe in a Helium Droplet, Oxford, Clarendon Press, 2003.

[11] Winkelmann C.B., Elbs J., Bunkov Y.M., Godfrin H, "Probing "cosmological" defects in superfluid ${ }^{3} \mathrm{He}-\mathrm{B}$ with a vibrating-wire resonator,” Physical Review Letters, 96 (20), 205301, May 2006.

[12] Boldyreva L.B, "The cavity structure effect in medicine: the physical aspect," Forschende Komplementärmedizin/Research in Complementary Medicine, 20, 322-326, 2013.

[13] Boldyreva L.B, "An analogy between effects of ultra low doses of biologically active substances on biological objects and properties of spin supercurrents in superfluid ${ }^{3} \mathrm{He}-\mathrm{B}$," Homeopathy, 100, issue 3, 187-193, 2011.

[14] Boldyreva L.B., Boldyreva E.M, "The Model of Superfluid Physical Vacuum as a Basis for Explanation of Efficacy of Highly Diluted Homeopathic Remedies," Homeopathy \& Ayurvedic Medicine, 1, issue 2, 2012.

[15] Boldyreva L.B, "The Physical Aspect of Action of Biologically Active Substances in Ultra-Low Doses and Low-Intensity Physical Factors on Biological Objects: Spin Supercurrents," Alternative and Integrative Medicine, 2, issue 3, 1000110 (6 pp.), 2013.

[16] Boldyreva, L.B, "The Physical Aspect of the Effects of Metal Nanoparticles on Biological Systems. Spin Supercurrents," Nanomaterials and Nanosciences.

[17] Boldyreva L.B, What does this give to physics: attributing the properties of superfluid ${ }^{3} \mathrm{He}-\mathrm{B}$ to physical vacuum? Moscow, KRASAND, 2012. 
[18] Mineev V.P, "Superfluid ${ }^{3}$ He: introduction to the subject," Sov. Physics Uspekhi, 26 (2), 160-175, 1983.

[19] Salomaa M., Volovik G.E, "Quantized vortices in superfluid ${ }^{3} \mathrm{He}$," Reviews of Modern Physics, 59, 533, 1987.

[20] Borovic-Romanov A.S., Bunkov Yu.M., Dmitriev V.V., Mukharskii Yu.M., Sergatskov D.A, "Investigation of Spin Supercurrents in ${ }^{3} \mathrm{He}-B$,” Physical Review Letters, 62, No. 14, 1631, 1989.

[21] Dmitriev V.V., Fomin I.A, "Homogeneously precessing domain in ${ }^{3}$ He-B: formation and properties," Journal of Physics: Condensed Matter 21, No. 16, 164202, 2009.

[22] Bunkov Yu.M, "Spin Superfluidity and Coherent Spin Precession," Journal of Physics: Condensed Matter, 21, No. 16, 164201 (6 pp), 2009.
[23] Sedov L.I, A Course in Continuum Mechanics, v. 1-4, WoltersNoordhoff, 1971-1972.

[24] Boldyreva L.B., Sotina N.B, "Superfliud Vacuum with Intrinsic Degrees of Freedom,” Physics Essays 5, 510-513, 1992.

[25] Purcell E.M, Electricity and Magnetism. Berkeley physics course, v. 2, McGraw-Hill Book company, 1965.

[26] Puttermann S, Superfluid Hydrodynamics, New York, 1974.

[27] Boldyreva L.B., Sotina, N.B, “'Hydden' dynamics in relativistic kinematics," Physics Essays, 16, No. 3, 2003.

[28] Boldyreva L.B, "Quantum correlations-Spin supercurrents," International Journal of Quantum Information, 12, No. 1, 1450007 (13 pp.), 2014.

[29] Boldyreva L.B, "The Wave Properties of Matter. The Physical Aspect," International Journal of Physics, 2, No. 6, 189-196, 2014. 